PROCEEDINGS OF THE

AMERICAN MATHEMATICAL SOCIETY

Volume 126, Number 12, December 1998, Pages 3633-3637

S $0002-9939(98) 04517-1$

\title{
BANACH SPACES FAILING THE ALMOST ISOMETRIC UNIVERSAL EXTENSION PROPERTY
}

\author{
D. M. SPEEGLE
}

(Communicated by Dale Alspach)

\begin{abstract}
If $X$ is an infinite dimensional, separable, uniformly smooth Banach space, then there is an $\epsilon>0$, a Banach space $Y$ containing $X$ as a closed subspace and a norm one map $T$ from $X$ to a $C(K)$ space which does not extend to an operator $\tilde{T}$ from $Y$ to $C(K)$ with $\|\tilde{T}\| \leq 1+\epsilon$.
\end{abstract}

A pair of Banach spaces $(E, X)$ with $E$ a closed subspace of $X$ is said to have the $\lambda$-into- $C(K)$ extension property ( $\lambda$-EP for short) if for every $C(K)$ space, and every linear map $T: E \rightarrow C(K)$, there is an extension $\tilde{T}: X \rightarrow C(K)$ of $T$ such that $\|\tilde{T}\| \leq \lambda\|T\|$. We will say that a separable space $E$ has the $\lambda$-universal extension property $(\lambda$-UEP) if $(E, X)$ has the $\lambda$-EP whenever $E$ imbeds as a (closed) subspace of a separable space $X$. We restrict to separable $X$ to rule out known counterexamples, namely $X=\ell_{\infty}$.

The best known result along these lines is Sobczyk's theorem [S], which in this language says that $c_{0}$ has the 2-UEP. In addition, Lindenstrauss and Pelczynski $[\mathrm{LP}]$ proved that whenever $E$ is a closed subspace of $c_{0},\left(E, c_{0}\right)$ has the $(1+\epsilon)$-EP for all $\epsilon>0$, a fact which was generalized to $\left(E, c_{0}(\Gamma)\right)$ by Johnson and Zippin [JZ]. Since $c_{0}$ is separably injective, it follows that any subspace $E$ of $c_{0}$ has the $(2+\epsilon)$-UEP for all $\epsilon>0$. (It is not hard to show that, in fact, if $(E, X)$ has the $M$-EP and $X$ has the $N$-UEP, then $E$ has the $N M$-UEP; see below.)

Turning now to spaces which have the 1-UEP, we see that the situation is completely solved. A result of Kalman's implies (cf. $[\mathrm{K}],[\mathrm{L}]$ ) that if the dual ball of a finite dimensional Banach space $E$ has only finitely many extreme points, then $E$ has the 1-UEP. In [L], Lindenstrauss proved the converse; namely, if a Banach space has the 1-UEP, then the dual ball of $E$ can have only finitely many extreme points.

However, the almost isometric case is still open. It is easy to see from Kalman's result that all finite dimensional spaces have the $(1+\epsilon)$-UEP for all $\epsilon>0$, which is the only positive result in this direction to date.

In this paper we investigate whether finite dimensional spaces are the only spaces which have the $(1+\epsilon)$-UEP for all $\epsilon>0$, which we refer to as the almost isometric universal extension property (AIUEP). Using a technique similar to one in [L], we

Received by the editors December 23, 1996 and, in revised form, April 25, 1997.

1991 Mathematics Subject Classification. Primary 46B20.

The author was supported in part by the NSF through the Workshop in Linear Analysis and Probability at Texas A\&M. 
are able to imbed some Banach spaces into the continuous functions on a weak*closed subset of its dual ball in such a way that this pair fails the $(1+\epsilon)$-EP. In particular, we get that $c_{0}$ and all infinite dimensional, separable, uniformly smooth spaces fail the AIUEP.

We begin by recalling that there is a correspondence between norm-one maps from a Banach space $\mathrm{E}$ to a $C(K)$ space and continuous functions from $K$ to the unit ball $\left(B_{E^{*}}, w^{*}\right)$. The application of this fact in the context of the extension property yields the following proposition, which can be found in [Z].

Proposition $1([\mathrm{Z}])$. Let $E$ be a closed subspace of the Banach space $X$, and let $\lambda \geq 1$. Then $(E, X)$ has the $\lambda$-EP if and only if there is a weak $k^{*}-w e a k^{*}$ continuous function $f: B_{E^{*}} \rightarrow \lambda B_{X^{*}}$ which extends functionals, i.e. $\left.f\left(e^{*}\right)\right|_{E}=e^{*}$.

A minor modificaton of Proposition 1 gives a simple necessary condition for the pair $(E, X)$ to have the $\lambda$-EP.

Proposition 2. Let $E$ be a closed subspace of the Banach space $X$, and let $\lambda \in \mathbb{R}$. If $(E, X)$ has the $\lambda$-EP, then every weak ${ }^{*}$ null sequence $\left\{e_{n}^{*}\right\}$ in $B_{E^{*}}$ has a weak* null sequence $\left\{x_{n}^{*}\right\}$ of extensions to $X^{*}$ satisfying $\left\|x_{n}^{*}\right\| \leq \lambda$.

Proof. By Proposition 1, $E \subset X$ has the $\lambda$-EP if and only if there is a weak*-weak* continuous map $f: B_{E^{*}} \rightarrow \lambda B_{X^{*}}$ which extends functionals.

Now, notice that if $f$ is such a function, so is $g\left(e^{*}\right)=\frac{f\left(e^{*}\right)-f\left(-e^{*}\right)}{2}$. In particular, there is such a function satisfying $g\left(e^{*}\right)=-g\left(-e^{*}\right)$. Now, suppose $E \subset X$ has the $\lambda$-EP, and let $e_{n}^{*} \rightarrow 0$. Then, $-e_{n}^{*} \rightarrow 0$ as well. So, $g\left(-e_{n}^{*}\right) \rightarrow g(0)$, and $g\left(-e_{n}^{*}\right)=-g\left(e_{n}^{*}\right) \rightarrow-g(0)$. Hence, $g(0)=0$, and in order for $g$ to be continuous, $\left\{g\left(e_{n}^{*}\right)\right\}_{n=1}^{\infty}$ is weak* null.

We are now ready to give a condition which implies that a Banach space $E$ fails the AIUEP. Recall that a weak slice of the unit ball $B_{E^{*}}$ is a set $S(e, \alpha)=\left\{e^{*} \in\right.$ $\left.B_{E^{*}}: e^{*}(e)>\alpha\right\}$. We say a Banach space has property $\mathcal{A}$ with constant $\alpha$ if there is a normalized weak ${ }^{*}$ null sequence $\left\{e_{n}^{*}\right\}$, an $\alpha \in[0,1)$, and a normalized sequence $\left\{e_{n}\right\} \in E$ such that $(1) e_{n}^{*}\left(e_{n}\right) \rightarrow 1$ and $(2) S\left(e_{n}, \alpha\right) \cap-S\left(e_{m}, \alpha\right)=\emptyset$ for all $n, m$.

Theorem 3. Let $E$ be a Banach space which satisfies property $\mathcal{A}$ with constant $\alpha$, and let $\epsilon<\frac{1-\alpha}{1+\alpha}$. Then, $E$ does not have the $(1+\epsilon)$ universal extension property. Moreover, there is a space $X$ containing $E$ as a codimension one subspace such that $(E, X)$ fails the $(1+\epsilon)-E P$.

Proof. Let $\left\{e_{n}^{*}\right\}$ and $\left\{e_{n}\right\}$ be sequences satisfying the conditions of property $\mathcal{A}$. Let $K=B_{E^{*}} \backslash\left(\bigcup_{n=1}^{\infty} S\left(e_{n}, \alpha\right)\right)$. We will show that $(E, C(K))$ does not have the $(1+\epsilon)$ extension property. Consider the map $E \rightarrow C\left(K, w^{*}\right)$ under the natural map $e \rightarrow \hat{e}$, where $\hat{e}\left(e^{*}\right)=e^{*}(e)$.

The first step is to verify that the natural map is an isometric imbedding. Since $K$ is weak ${ }^{*}$ closed, it suffices to show that the weak* closed symmetric convex hull of $K$ is all of $B_{E^{*}}$. To do this, it suffices then to show that the symmetric hull of $K$ contains $S_{E^{*}}$. So, suppose $e^{*}$ is a norm one functional in $E^{*}$ which is not in $K$. Then, $e^{*}$ is in $S_{n}$ for some $n$. So, by property $\mathcal{A},-e^{*}$ is not in $S_{m}$ for any $m$ and $K \cup-K$ contains $S_{E^{*}}$.

To show that $E$ fails the almost isometric universal extension property, we will show that $E$ together with the above imbedding into $C(K)$ fails the conditions of Proposition 2. To this end, let $\mu_{n}$ be extensions of $e_{n}^{*}$ to functionals on $C(K)$ with 
norm less than $1+\epsilon$, and let $f: K \rightarrow \mathbb{R}$ be the constant one function. We show that $\mu_{n}(f)$ is bounded away from 0 .

Write $\mu_{n}$ as $\mu_{n}^{+}-\mu_{n}^{-}$via the Hahn decomposition $[\mathrm{R}]$. Then, for some $\beta_{n} \geq 0$,

$$
\begin{aligned}
1-\beta_{n} & =\int_{K} \hat{e}_{n} d \mu_{n} \\
& \leq \int_{K_{1}^{n} \cup K_{2}^{n}} \alpha d \mu_{n}^{+}+\int_{K_{1}^{n} \cup K_{2}^{n}} d \mu_{n}^{-} \\
& =\alpha \mu_{n}^{+}\left(K_{1}^{n}\right)+\mu_{n}^{-}\left(K_{2}^{n}\right),
\end{aligned}
$$

where $K_{1}^{n}$ and $K_{2}^{n}$ correspond to the positive and negative parts of $\mu_{n}$ on $K$.

We also have, by the norm of $\mu_{n}$, that

$$
\mu_{n}^{+}\left(K_{1}^{n}\right)+\mu_{n}^{-}\left(K_{2}^{n}\right) \leq 1+\epsilon .
$$

Adding $\frac{-2}{1-\alpha}$ times (1) to $\frac{1+\alpha}{1-\alpha}$ times (2), we can compute

$$
\begin{aligned}
\mu_{n}(f) & =\int_{K} f d \mu_{n} \\
& =\mu_{n}^{+}\left(K_{1}^{n}\right)-\mu_{n}^{-}\left(K_{2}^{n}\right) \\
& =\frac{-2}{1-\alpha}\left(\alpha \mu_{n}^{+}\left(K_{1}^{n}\right)+\mu_{n}^{-}\left(K_{2}^{n}\right)\right)+\frac{1+\alpha}{1-\alpha}\left(\mu_{n}^{+}\left(K_{1}^{n}\right)+\mu_{n}^{-}\left(K_{2}^{n}\right)\right) \\
& \leq \frac{-2}{1-\alpha}\left(1-\beta_{n}\right)+\frac{1+\alpha}{1-\alpha}(1+\epsilon) \\
& =-1+\frac{1+\alpha}{1-\alpha} \epsilon+\frac{2 \beta_{n}}{1-\alpha} .
\end{aligned}
$$

So, since $\beta_{n} \rightarrow 0$ and $\epsilon<\frac{1-\alpha}{1+\alpha}$, we have that for large $n, \mu_{n}(f) \leq c<0$ for some constant $c$, as desired.

To prove the last statement, instead of looking at $E \rightarrow C(K)$, we could just as well have considered $E$ imbedded into $\overline{s p}(E \cup\{f\})$ in $C(K)$, and the proof goes through. Thus, $E$ imbeds as a codimension one subspace of a space $X$ such that $(E, X)$ fails the almost isometric universal extension property.

By examining the proof of Theorem 3 more carefully, we gain the following additional information. Since weak* null sequences in $B_{E^{*}}$ correspond to maps from $E$ to $c_{0}$, it follows that spaces with property $\mathcal{A}$ actually fail the $(1+\epsilon)$-into- $c_{0}$ extension property. Of course, by Sobczyk's theorem [S], every norm-one map from a subspace of a separable space into $c_{0}$ extends to a map of norm less than or equal to 2 .

Proposition 4. If $X$ is an infinite dimensional, separable, uniformly smooth Banach space, then $X$ has property $\mathcal{A}$ with constant $\alpha$ for some $\alpha \in[0,1)$.

Proof. We construct the necessary sequences $\left\{y_{n}^{*}\right\},\left\{y_{n}\right\}$, and the constant $\alpha$. Let $\left\{x_{n}\right\}$ be a sequence which is dense in $B_{X}$. Let $y_{1}^{*}$ be in the unit sphere $S_{X^{*}}$ of $B_{X^{*}}$, and let $y_{1}$ be a norm one element in $X$ such that $y_{1}^{*}\left(y_{1}\right)=1$. Let $S_{1}=\left\{x^{*} \in S_{X^{*}}\right.$ : $\left.\left|x^{*}\left(y_{1}\right)\right|<\frac{1}{2}, x^{*}\left(x_{1}\right)<\frac{1}{2}\right\}$. Then, $S_{1} \neq \emptyset$, so let $y_{2}^{*} \in S_{1}$, and let $y_{2} \in S_{X}$ norm $y_{2}^{*}$. Continuing in this fashion, we get $y_{i}^{*} \in S_{i}=\left\{x^{*} \in S_{X^{*}}:\left|x^{*}\left(y_{j}\right)\right|<\frac{1}{i+1}, x^{*}\left(x_{j}\right)<\right.$ $\frac{1}{i+1}$ for all $\left.1 \leq j<i\right\}$, where $y_{j} \in S_{X}$ satisfy $y_{j}^{*}\left(y_{j}\right)=1$. To define the constant $\alpha$, let $\delta$ denote the modulus of convexity of $X^{*}$. We claim that $\alpha=1-\delta\left(\frac{1}{4}\right)$ 
together with the above $\left\{y_{i}\right\}$ and $\left\{y_{i}^{*}\right\}$ satisfies the properties required for $X$ to have property $\mathcal{A}$. (Note that since $X^{*}$ is uniformly convex, $\alpha \in[0,1)$.)

To prove the claim, first note that since $\left\{x_{n}\right\}$ is dense in $B_{X}$, the sequence $\left\{y_{n}^{*}\right\}$ converges to 0 in the weak* topology. So, it remains to prove that $S\left(y_{i}, \alpha\right) \cap$ $-S\left(y_{j}, \alpha\right)=\emptyset$. For $i=j$, this is immediate, while for $j<i$, we have $\left\|y_{i}+y_{j}\right\| \geq$ $\left|y_{i}^{*}\left(y_{i}+y_{j}\right)\right| \geq 1-\frac{1}{i+1} \geq \frac{1}{2}$. We also have that the diameter of the slice $S\left(y_{i}, \alpha\right)$ is less than $\frac{1}{4}$. Hence, if $x \in S\left(y_{i}, \alpha\right) \cap-S\left(y_{j}, \alpha\right)$, it follows that $\left\|y_{i}-x\right\|<\frac{1}{4}$ and $\left\|x+y_{j}\right\|<\frac{1}{4}$; hence, $\left\|y_{i}+y_{j}\right\|<\frac{1}{2}$; therefore, $S\left(y_{i}, \alpha\right) \cap-S\left(y_{j}, \alpha\right)=\emptyset$.

We note here that the result of Theorem 3 is the best possible when $E=c_{0}$. Indeed, if we consider $e_{n}$ to be the standard unit vector basis for $c_{0}$, it is easy to see that, if we consider slices of the extreme points of $B_{\ell_{1}}$ rather than slices of $B_{\ell_{1}}$, then $c_{0}$ has property $\mathcal{A}$ with constant $\alpha$ for all $\alpha>0$; hence, $c_{0}$ fails the $(2-\epsilon)$-UEP for all $\epsilon>0$. (It is easy to see that, in fact, $\left(c_{0}, c\right)$ fails the $(2-\epsilon)$-EP for all $\epsilon>0$.) However, we do not know in general if finding the best possible $\alpha$ yields the best possible $\lambda$ for which $(E, X)$ has the $\lambda$-into- $c_{0}$ extension property, even when $E$ is assumed to be a codimension one subspace of $X$.

Finally, we prove the fact mentioned in the introduction.

Proposition 5. Let $X$ be a separable Banach space with the $N$-UEP, and let $(E, X)$ have the $M-E P$. Then, $E$ has the $M N-U E P$.

Proof. Let $T: E \rightarrow C(K)$ be a bounded linear operator, let $i: E \rightarrow X$ denote the imbedding of $E$ into $X$, and let $j: E \rightarrow Y$ denote an imbedding into an arbitrary separable Banach space $Y$. The proof follows from taking pushouts (cf. $[\mathrm{J}]$ for definitions and properties) as in the following commutative diagram. All of the maps along the outer commuting square are isometric imbeddings, and $P$ is separable.

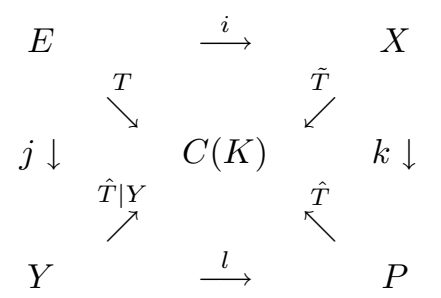

The map $\tilde{T}$ is an extension of $T$ to $X$ such that $\|\tilde{T}\| \leq M\|T\|$, and $\hat{T}$ is an extension of $\tilde{T}$ to $P$ such that $\|\hat{T}\| \leq N\|\tilde{T}\| \leq M N\|T\|$. The restriction of $\hat{T}$ to $Y$ is the desired extension of $T$ from $E$ to $Y$.

\section{ACKNOWLEDGMEnT}

The author would like to thank W. B. Johnson for suggesting improvements to the author's original argument. In particular, Proposition 2 was suggested by him.

\section{REFERENCES}

[J] W. B. Johnson, Extensions of $c_{0}$, Positivity (to appear).

[JZ] W. B. Johnson and M. Zippin, Extensions of operators from subspaces of $c_{0}(\gamma)$ into $C(K)$ spaces, Proc. AMS 107 (1989), 751-754. MR 90b:46045

[K] J. A. Kalman, Continuity and convexity of projections and barycentric coordinates in convex polyhedra, Pacific J. Math 22 (1961), 1017-1022. MR 24:A3557 
[L] J. Lindenstrauss, Extension of compact operators, Memoirs of the AMS 48 (1964). MR 31:3828

[LP] J. Lindenstrauss and A. Pelczynski, Contributions to the theory of classical Banach spaces, J. Functional Analysis 8 (1971), 225-249. MR 45:863

[R] H. L. Royden, Real Analysis 3rd Ed., Macmillan, New York, 1988. MR 90g:00004

[S] A. Sobczyk, Projection of the space $m$ on its subspace $c_{0}$, Bull. Amer. Math. Soc. 47 (1941), 938-947. MR 3:205f

[Z] M. Zippin, A global approach to certain operator extension problems, Lecture Notes in Math. 1470 (1991), 78-94. MR 93b:47011

Department of Mathematics, Texas A \& M University, College Station, Texas 77843

E-mail address: speegle@math.tamu.edu

Current address: Department of Mathematics, Saint Louis University, Saint Louis, Missouri 63103 